\title{
ILCEA
}

Revue de l'Institut des langues et cultures

d'Europe, Amérique, Afrique, Asie et Australie

$16 \mid 2012$

La culture progressiste à l'époque de la guerre froide

„Die Einheit von Kunst und Leben“ - eine totalitäre Utopie der politischen und künstlerischen Avantgarde. Die Kulturrevolution in der SU 1929 und der Bitterfelder Weg in der DDR $1959 \mathrm{im}$ Vergleich

"L'unité de l'art et de la vie» - une utopie totalitaire de l'avant-garde politique et artistique. Comparaison de la révolution culturelle en URSS en 1929 et de la voie de Bitterfeld en RDA en 1959

\section{Eckhard Gillen}

\section{(2) OpenEdition}

Journals

Édition électronique

URL : http://journals.openedition.org/ilcea/1287

DOI : 10.4000/ilcea.1287

ISSN : 2101-0609

Éditeur

UGA Éditions/Université Grenoble Alpes

Édition imprimée

ISBN : 978-2-84310-232-5

ISSN : 1639-6073

Référence électronique

Eckhard Gillen, „Die Einheit von Kunst und Leben“ - eine totalitäre Utopie der politischen und

künstlerischen Avantgarde. Die Kulturrevolution in der SU 1929 und der Bitterfelder Weg in der DDR 1959 im Vergleich“, ILCEA [Online], 16 | 2012, Online erschienen am: 04 Juli 2012, abgerufen am 22 März 2021. URL: http://journals.openedition.org/ilcea/1287 ; DOI: https://doi.org/10.4000/ilcea.1287

Ce document a été généré automatiquement le 22 mars 2021

(C) ILCEA 


\title{
„Die Einheit von Kunst und Leben“ - eine totalitäre Utopie der politischen und künstlerischen Avantgarde. Die Kulturrevolution in der SU 1929 und der Bitterfelder Weg in der DDR 1959 im Vergleich
}

\author{
"L'unité de l'art et de la vie»- une utopie totalitaire de l'avant-garde politique \\ et artistique. Comparaison de la révolution culturelle en URSS en 1929 et de la \\ voie de Bitterfeld en RDA en 1959
}

Eckhard Gillen

Es geht in diesem Vortrag nicht um Kunst um der Kunst willen (l'art pur l'art), sondern um Kunst als Medium einer Utopie, als Manifest einer neuen Weltordnung oder als Ausdruck einer Ideologie. Ist die Umwandlung der alten Gesellschaft in die neue harmonische Menschengemeinschaft gelungen, verliert die Kunst ihre Erziehungsfunktion als Leitbild während der Transformation und geht im neuen Leben auf.

2 In der Debatte nach dem Fall der Mauer um den Wert bzw. Unwert der Kunst in der DDR wird dieser Kunst im Auftrag einer Idee oder Ideologie generell jeder Kunstwert abgesprochen. Siegfried Gohr als Direktor des Museum Ludwig in Köln verweigerte ihr den Auftritt im Museum sehr zum Unwillen des Sammlers Peter Ludwig. Der gleiche Direktor schätzte und würdigte dagegen die Bestände der sowjetischen Avantgarde als autonome Kunstwerke, obwohl auch sie Modelle einer neuen Welt und eines neuen Menschen sind. Die weltweite Präsenz dieser russisch-sowjetischen Avantgarde in den Museen der Moderne ist, so Boris Groys, kein Beleg für ihren historischen Sieg, sondern für ihre historische Niederlage: „Unsere Museen sind eben die Orte, wo Glauben und 
Hoffnungen von damals auf die wirkliche Transformation der Welt ihre endgültige Ruhe finden." ${ }^{11}$ Das zeitweise Bündnis der künstlerischen Avantgarde mit totalitären Ideen zur Erlösung der Menschheit wird so von den puristischen Wächtern der reinen Kunst immer noch verdrängt.

Mit dem Programm der „I. Bitterfelder Konferenz“ im April 1959 im Kulturhaus auf dem Gelände des VEB Elektrochemisches Kombinat Bitterfeld übernimmt auch die SED für die DDR die sowjetrussische Idee der Kulturrevolution: die Herstellung der „Einheit von Kunst und Leben“. Man glaubte bereits 1960, „die Trennung von Kunst und Leben bei uns in der DDR ein für allemal“ überwinden zu können. ${ }^{2}$ Die Künstler sollten als Zirkelleiter die Arbeiter künstlerisch ausbilden, um sie so im Sinne des „sozialistischen Humanismus“ zu „,neuen Menschen“, die alle ihre Fähigkeiten entfalten können, zu machen.

4 Für das Diplom, das ihn für staatliche Aufträge qualifiziert, schafft der heutige Weltstar des kapitalistischen Kunstmarktes Gerhard Richter mit dem Wandbild „Lebensfreude“ im Deutschen Hygienemuseum Dresden 1956 ein Musterbild des Sozialistischen Realismus. Paradiesische Szenen vom Leben im Kommunismus münden in der Darstellung einer sozialistischen Familie über der Ausgangstür: Die Mutter mit ihrem Kind in der Pose der säkularisierten Madonna und der durch einen Traktor als Werktätiger auf dem Land gekennzeichnete Vater werden von Friedenstauben umflattert. ${ }^{3}$ Richters Wandbild wirkt wie eine Illustration von Maxim Gorkis berühmter Rede 1934 auf dem I. Allunionskongress der Sowjetschriftsteller, in der die Idee des Sozialistischen Realismus zum ersten Mal öffentlich vorgestellt wurde. „Der Sozialistische Realismus bejaht das Dasein [...] für das große Glück, auf der Erde zu leben, die der Mensch [...] in eine schöne Wohnstätte der zu einer Familie vereinigten Menschheit verwandeln will."“4

5 Zur Ideengeschichte der sowjetischen Kulturrevolution gehört nicht nur der Bitterfelder Weg in der DDR, sondern auch die Art und Weise, wie die totalitäre Parole der Einheit von Kunst und Leben von Teilen der westdeutschen Studentenbewegung übernommen worden ist. Ein Jahrzehnt nach der Kulturkonferenz der SED eröffnet z.B. Dieter Hacker $1971 \mathrm{im}$ eingemauerten Westberlin die "7. Produzentengalerie“ mit dem Appell Tötet Euren Galeristen. Kollegen! Gründet Eure eigene Galerie. Gründet eine Produzentengalerie auf einem Plakat von 1971. Im Sinne der kommunistischen Kulturrevolution und im Geiste der sich in viele konkurrierende Mini-Parteien und dogmatische Politsekten atomisierenden Studentenbewegung kritisiert auch er als politisch bewusster Künstler des Westens die Kunst als kleinbürgerlich-individuelle Produktion von Werken, die in Galerien verkauft werden. Die Kunst soll dem Volke dienen. Jörg Immendorff gründet 1970 eine „Rote Zelle Kunst“, verlässt die BeuysKlasse, wird Kunstlehrer an einer Hauptschule, schließt sich einer maoistischen Sekte an und porträtiert sich 1973 im Agitpropstil. „Ich wollte Künstler werden“ ist eine gemalte Selbstkritik: „Ich träumte davon, in der Zeitung zu stehen, von vielen Ausstellungen, und natürlich wollte ich etwas ,Neues' in der Kunst machen. Mein Leitfaden war der Egoismus."

6 Unter den Schülern von Joseph Beuys bewegt sich Imi Knoebel auf den Spuren der Anfang der sechziger Jahre in Ost- und Westdeutschland völlig vergessenen russischen Avantgarde, wobei er sich ausdrücklich auf Kasimir Malewitsch bezieht: „Damals kam gerade dieses Buch raus Die gegenstandslose Welt. Fasziniert waren wir von dem Schwarzen Quadrat. [...] Keine Sau kannte den. Und das war immer, was wir vor uns 
hergeschoben hatten, also die russische Bewegung. Nicht umsonst dann unsere Kahlköpfe." ${ }^{\text {"An }}$ An sich selbst vollzogen sie mit der Rasur des Schädels die totale Entleerung der Leinwand.

7 Das Meister-Schüler-Modell, das die Fotografie von Kasimir Malewitsch mit seinem Schüler Gustav Klucis zeigt, wird auch von Joseph Beuys und seinem Schüler Palermo 1965 in der Kunstakademie Düsseldorf zelebriert.

8 Malewitsch ist jedenfalls parallel zur Oktoberrevolution im Bereich der Ästhetik Anstifter eines radikalen Neuanfangs, der, wie Lenin im Bereich der Gesellschaftspolitik, künstlerisch Tabula rasa machen will. Als Anarchist lehnt er jede Form des Staates ab und deklarierte 1918 die Suprematie des Ich, die das materielle Sein souverän ignorierte. Mit Lenin und den Bolschewiki verband ihn der revolutionäre Impetus, die Welt nach seinem Willen neu zu erschaffen. Er sah eine Analogie zwischen dem revolutionären "Zerbrechen“ des Staates (das auch Lenin wollte) und seiner Vorstellung vom notwendigen Absterben der materiellen Wirklichkeit: „wir erklären alle Dinge für nicht existent. “7

Zum Symbolbild für diesen Paradigmenwechsel von der Repräsentation zur radikalen Konstruktion einer neuen Welt, den die Futuristen und Suprematisten in Russland bzw. der Sowjetunion vollzogen, wurde das „Schwarzes Quadrat“ (um 1914/15). Malewitsch und seine Schüler proklamierten die Zerstörung der alten Welt und ihrer Werte, um aus ihren Trümmern eine vollständig neue, gereinigte und bessere Welt zu erschaffen. Die künstlerische Avantgardeglaubte, die politische Avantgarde auf Zeit benutzen zu können, um ihrer Utopie einen konkreten Ort in der Wirklichkeit zu schaffen.

In den Fabriken gibt es weder „Dunst, noch Ruß, noch Gestank, noch Staub. In der reinen, frischen Luft arbeiteten die Menschen kraftvoll und gleichmäßig, das Licht war nicht schmerzlich grell, doch drang es überall hin. [...] Hebel, stählernen Riesenhänden ähnlich, bewegten sich gleichmäßig und glatt [...]“. Diese Sätze lesen sich wie eine Beschreibung des Gemäldes „Textilarbeiterinnen“ von Alexander Deinekaaus dem Jahr 1927, auf dem junge Frauen barfuss in luftigen Sommerkleidern mit grazilen Bewegungen in einem sonnendurchfluteten, staubfreien Raum die automatisierte Textilproduktion kontrollieren. Sie finden sich in dem 1908 erschienen Roman „Der rote Stern ${ }^{\text {“8 }}$ von Alexander A. Bogdanow. ${ }^{9}$ Der russische Revolutionär Leonid wird von einer auf der Erde gelandeten Delegation der Marszivilisation eingeladen zu einem längeren Aufenthalt auf ihrem Planeten, um ihre „Lebensordnung“ kennenzulernen. Wie sich schnell herausstellt, ist auf dem Mars alles, was auf der Erde noch revolutionäre Theorie ist, bereits vernünftig und planmäßig organisierte Wirklichkeit. Auch die kommunistische Marszivilisation basiert, wie die spätere Sowjetunion, auf einer vergangenen heroischen Zeit, in der unter dem Kommando eines Chef-Ingenieurs mit strenger Energie, „kalter Entschlossenheit, ja fast Grausamkeit“ der Bau eines Systems von großen Kanalbauten, an denen hunderttausende Menschen arbeiteten, vorangetrieben worden war. (S. 63) Bereits 1908 werden die gigantischen Kanalprojekte der Stalinzeit von Bogdanow antizipiert.

11 Da die Ressourcen des Mars bald erschöpft sein werden, planen die kommunistischen Marsmenschen die Kolonisierung anderer Planeten. Auf ihrem Nachbarplaneten beobachten sie mit Interesse die Aktivitäten der sozialistischen Bewegungen, doch „Ihr Sozialismus ist noch lange nicht unser Sozialismus"(S. 138).Der kalte, analysierende Verstand des Astronomen und Mathematikers Sterni, der stets „alle Phantasien des Geistes und des Gefühls“ (S. 123) zerstört, kommt mit messerscharfer Logik zu dem 
Schluss: „die Kolonisierung der Erde fordert die völlige Ausrottung der Erdenmenschen.“ Die einzige Alternative wäre „der sofortige Versuch einer sozialistischen Erziehung der Erdenmenschheit [...]“ (S. 135). Kann die vollständige Umerziehung nicht durchgeführt werden, so bleibt dem Erzieher nur die Liquidierung: „Ich rede von der Ausrottung der ganzen Erdenmenschheit [...] Das höhere Leben darf nicht dem niedern geopfert werden. [...] Es bedeutet daher keinen Verlust, wenn sich auf der Erde [...] schon heute unser Sozialismus verwirklicht, das unvergleichlich harmonischere Leben mit seiner ununterbrochenen, unbesieglichen Entwicklung." (S. 139f.)

12 In seinem Roman stellt Bogdanow die entscheidende Lebensfrage: entweder gelingt die Überwindung der Anarchie der sozialen Kräfte und Interessen durch die Revolution oder es kommt zum „Zerfall der Zivilisation“. ${ }^{10}$ Auch Leo Trotzkij war überzeugt, dass die Revolution nur mit "Gewalt, Ausrottung und Zerstörung“ die Voraussetzungen für die neue Gesellschaft schaffen kann. „Der Umsturz rettet die Gesellschaft und die Kultur, aber mit den Methoden der grausamsten Chirurgie. [...] alles, was stört, wird mitleidlos niedergetrampelt. “"

Ökonomie und Gesellschaft waren nach der Oktoberrevolution im Bürgerkrieg in den Zustand des einfachen Tauschhandels und eines Krieges aller gegen alle zurückgefallen: Die besten Voraussetzungen für die Verwirklichung einer radikalen „Umwertung der Werte“ “.12 Ganz im Sinne Nietzsches glaubte Trotzkj, dass die Revolution „unermüdlich die Auslese aus allem, was es in der Arbeiterklasse an Bestem, Unerschrockenstem und Opferbereitestem gibt", treffen wird..$^{13}$ Im Zuge seiner Höherentwicklung wird der Mensch mit Hilfe der Technik wirklich Berge versetzen, die Erde umbauen, „die Richtung der Flüsse ändern und den Ozeanen Regeln vorschreiben. [...] Die kommunistische Daseinsform wird [...] bewußt aufgebaut, durch die Idee überprüft, ausgerichtet und korrigiert werden". ${ }^{14}$ Schließlich wird der Mensch sich selbst umbauen und harmonisieren. „Der Mensch wird [...] mit seinem Willen bis in die letzten Tiefen seines Unbewußten vorzudringen und sich so auf eine Stufe zu erheben einen höheren gesellschaftlich-biologischen Typus, und wenn man will - den Übermenschen zu schaffen. ${ }^{15}$ Die Revolution verspricht eine letzte Schlacht, den totalen Krieg für den totalen Frieden einer auf ewig befriedeten Gesellschaft, den Zustand der „Entropie“ als glücklich austariertes Gleichgewicht nach dem Energieschub der Revolution. Bogdanow entfaltet in seinem Roman die statische Utopie eines zeitlosen Zustandes ewiger Glückseligkeit nach dem Ende der Geschichte. Stalin war es, der nach Lenins Tod die Weltrevolution zugunsten eines „Sozialismus in einem Land“ aufgab. Die letzte, endgültige Säuberung sollte „Der Große Terror“ 1936-1938 werden. ${ }^{16}$ Nadeschda Mandelstam beschreibt in ihren Memoiren den Zustand einer „Utopie als Ordnungsentwurf“" ${ }^{17}$ die ewige Glückseligkeit, Stabilität, Harmonie und damit zugleich auch Stillstandals soziale Konstruktion: Es schien so, als sei „die Zeit eingefroren, sei stehengeblieben. [...] Man hatte uns eingeredet, in unserem Land würde sich niemals mehr etwas ändern und die übrige Welt müsse nur bis zu unserem Zustand gelangen, das heißt auch in die neue Ära eintreten, dann gäbe es niemals mehr eine Veränderung “. ${ }^{18}$

Die Kunst in der Periode des Stalinismus der 30er Jahre sollte daher der Widerspiegelung der vollkommenen Harmonie der Gesellschaft als Gesamtkunstwerk dienen. Diesen Wandel von der revolutionären Eruption zur Erstarrung zeigen die 
beiden Gemälde von Konstantin Juon aus der Tretjakow Galerie: „Der neue Planet“, 1921, und „Parade. November 1941“, 1949.

Die utopischen Romane und Theorien von Alexander A. Bogdanow entwarfen schon lange vor der Revolution die totalitäre Utopie einer entdifferenzierten, homogenen Gesellschaft (z.B. durch Zwangskollektivierung) als regressives Gegenmodell zum westlichen Prinzip zunehmender gesellschaftlichen Ausdifferenzierung und Arbeitsteilung. Mit seiner „Allgemeinen Organisationslehre“ ${ }^{\text {“19 }}$ entwickelte Bogdanow die Theorie einer kollektivistischen Gesellschaft ${ }^{20}$ der Zukunft, die auf der Idee einer harmonischen Organisiertheit der Dinge vermittels der Technik und der Menschen über die Wissenschaften aufbaute und zum Vorbild wurde für Lenins Vorstellung der sozialistischen Gesellschaft als Fabrik. Der Arbeitswissenschaftler und Proletkultdichter Alexej Gastew, Direktor eines 1920 in Moskau gegründeten „Zentralen Arbeitsinstitutes", verherrlichte seit 1918 in literarischen Werken und Artikeln wie „Poesie des Arbeiterschlages“ (1918) die ,unio mystica' der Maschinen mit der Psyche der Arbeiter. Auf der Organisationslehre von Bogdanow aufbauend, übertrug dieser Exponent des russischen Taylorismus die Verfahren der „Wissenschaftlichen Arbeitsorganisation“ (russisch NOT abgekürzt) auf alle Bereiche des Alltagslebens. Die ganze Gesellschaft soll nach den Prinzipien der maschinellen Technik und Fabrikdisziplin organisiert werden.

Die sowjetischen Avantgarde-Künstler, vor allem die Konstruktivisten, adaptierten diese mechanischen Prinzipien, zum Beispiel führte Rodtschenko die NOT als Studienfach an der Metallwerkstatt der WChUTEMAS (Höhere Staatliche KünstlerischTechnische Werkstätten 1921-1926) ein und erhob im gemeinsam mit seiner Frau Warwara Stepanowa formulierten „Produktivistenmanifest“ Bogdanows Begriff der „Tektonik“ zur zentralen Kategorie im Sinne einer Gleichsetzung von Konstruktion mit Organisation. ${ }^{21}$ Als Spezialisten der Materialorganisation fühlten sich die Konstruktivisten sowohl in der Lage, den ,Widerstand des Materials ${ }^{\star}$ zu brechen, es sich "gefügig zu machen und ihm jede gewünschte Form zu geben“ (Boris Groys) als auch im übertragenen Sinne das ,Menschenmaterial ${ }^{*}$ in den Fabriken psychophysisch zu erziehen. In ihrer Einbildungskraft glaubten sie dank ihres Organisationswissens auch über die materielle und soziale Wirklichkeit zu verfügen. „Die ,Tektologie‘ räumte dem Künstler als aktivem Organisator des Bewußtseins und der Emotionen der Massen, ihrer sogenannten ,Psychoideologie‘ und ihrer ,materiellen Kultur', einen zentralen Platz beim Aufbau der neuen Gesellschaft ein." ${ }^{22}$ Bogdanows Organisationswissenschaft zielt auf die Aufhebung der Gegensätze, Widersprüche und des Individualismus zugunsten des absoluten Gleichgewichts, der Homogenität und des Kollektivismus.Bogdanow und die Vertreter der von ihm initiierten ,Proletkult'Bewegung betrachteten die Kunst als ein Medium zur Organisation sozialer Erfahrung. 23

17 Die Vertreter der sowjetischen Avantgarde einte bei aller Differenz in Fragen der formalen Organisation die Überzeugung, dass der Künstler im Sinne einer „Produktionskunst“ die neue Arbeitsgesellschaft aktiv gestalten muss (Design, Architektur, Städtebau) und durch eine massenwirksame Kunst (Wandbild, Plakat, Zeitschriften) die Bewusstseinsbildung der Arbeiter (die „Künstler als Ingenieure der Seele“ nach dem berühmten Diktum von Stalin) beeinflussen sollte. „Nicht das Hervorbringen neuer Bilder [...], sondern die Formung eines neuen Menschen unter 
Nutzung der Kunst als eines der Produktionsmittel war der Kompaß des Futurismus von seinen Kindertagen an.“" ${ }^{24}$

18 Jewgenij Samjatin schreibt aufgrund seiner politischen Erfahrungen und Enttäuschungen $^{25}$ mit seinen Roman „Wir“ ${ }^{26}$ eine hellsichtige Antiutopie als Gegenentwurf zum mechanisch-totalitären Sozialismusmodell in Bogdanows utopischen Romanen. ${ }^{27}$ Wir lesen die Tagebucheintragungen der Nummer D-503, des Konstrukteurs des Raumschiffes „Integral“ und Mathematikers des „Einzigen Staates“, der nach einem 200jährigen Krieg zwischen Stadt und Land übriggeblieben ist und sich mit einer grünen, gläsernen Mauer vom unzivilisierten Rest der Welt abschottet (Sozialismus in einem Land!). Seine Bewohner sind Nummern, alle haben die gleichen Gedanken. Mit der Fertigstellung des Raumschiffes wartet auf diese Nummern die Aufgabe, ,jene unbekannten Wesen, die auf anderen Planeten - vielleicht auch in dem unzivilisierten Zustand der Freiheit - leben, unter das segenreiche Joch der Vernunft $\mathrm{zu}$ beugen. Sollten sie nicht begreifen, daß wir ihnen das mathematisch-fehlerfreies Glück bringen, haben wir die Pflicht, sie zu einem glücklichen Leben zu zwingen. [...] Wir werden die wilde, krumme Linie geradebiegen [...] Denn die Linie des Einzigen Staates ist die Gerade“ (S. 5).

Während Andrej Gastev sich für das "Geradebiegen des Volkes“ in „Schulen der Arbeitsbewegungen“ ausspricht, ${ }^{28}$ überträgt Rodtschenko dieses psychotechnische Denken auf die Kunst. In seinem Manifest „Die Linie“ (1921) behauptet er „den totalen Sieg“ der Linie über „die letzten Bastionen der Malerei“". ${ }^{29}$ Die geometrisch objektive Linie als Basiselement für Mathematiker und Konstrukteure galt ihm und den Konstruktivisten als Garant gegen mögliche Rückfälle in die bourgeoise Kunst der Stimmungswerte und Gefühle, die als Ausdruck subjektiver Empfindungen und Phantasien von der Avantgarde vehement abgelehnt wurde. Die Diagonale wird zur wichtigsten, Dynamik suggerierenden Organisationsfigur der Konstruktion, mit der das Menschenmaterial, bzw. die Natur symbolisch unterworfen wird. Beispiele dafür sind der T-Träger auf Gustavs Klucis Fotocollage „Dynamische Stadt“ (1919)als die stützende Schrägachse für die gesamte Bildkonstruktion, und die Lenin gewidmete Rednertribüne von Lissitzky. Die geometrischen Grundformen Kreis, Kubus, Zylinder und ihr freies Schweben im Raum kommen noch aus dem Suprematismus, werden aber von Klucis in Verbindung mit den fotografischen Einsprengseln von Montagearbeitern, T-Trägern und Hochhäusern zu Metaphern für den Bau einer neuen Welt.

Das Plakat von N. Dolgorukow, „Die gewaltige Energie der Wolga soll der sozialistischen Rekonstruktion dienen!“ (1930), zeigt mit einem Staudamm, der aus der Vogelperspektive gesehen als weiße Diagonale von links unten nach rechts oben erscheint, den „Eingriff der Geometrie in die Geographie“ als Planung, dem der reale "Einbruch des Menschen in die Natur" folgen wird. ${ }^{30}$ Diese technokratische Vernunft garantiert, dass die Arbeiter „zu einem einzigen millionenhändigen Körper“ verschmelzen wie es das Plakat „Erfüllen wir den Plan“ von Gustav Klucis zeigt. Der Roman „Wir“ endet mit dem Satz: „Die Vernunft muß siegen!“

21 Mit der Entscheidung für den Ersten Fünfjahrplan im Frühjahr 1929, der die forcierte Industrialisierung einleitete, und für die totale Kollektivierung mit dem Ziel der „Liquidierung der Kulaken als Klasse“, hatte sich Stalin für eine bedingungslose Flucht nach vorn, ohne Möglichkeit der Umkehr entschlossen. Im Moment der von oben angeordneten zweiten und endgültigen Revolution, einem permanenten Krieg der Regierung gegen die eigene Bevölkerung, werden die Begriffe ausgetauscht: An die 
Stelle schematisierter, typisierter Funktionsträger rückt der „lebendige Mensch“. Die Parole vom „lebendigen Menschen“ bekommt in den 30er Jahren den Charakter einer Leitkultur gegen alles ,Mechanische', ,Logische` und ,Abstrakte`. Die gleiche Parole gegen Formalismus und Schematismus galt auch in der DDR, wie wir sehen werden. Die Theoretiker des Sozialistische Realismus wandten sich daher gegen schematische, abstrahierende Darstellungsweisen und forderten vom Künstler die Darstellung des blutvollen Lebens.

Als erste bekamen im Februar 1922 die Mitglieder der AChRR (Assoziation revolutionärer Künstler Russlands) den Rat vom ZK, in die Fabriken zu gehen, „um mit ihrer Kunst dem Volke zu dienen“. Sie gingen mit Block und Bleistift in die Betriebe „genau wie die Künstler aus Barbizon mit der Staffelei in die Wälder Fontainebleaus zogen [...] mit der vorsintflutlichen Ausrüstung einer Staffelei-Ästhetik, mit den weißen Handschuhen einer bürgerlichen Kunst" ${ }^{\text {" }}{ }^{31}$ notiert Boris Arvatov, Theoretiker der Produktionskunst. Er mokiert sich über die "gourmethafte Erregung“ eines AChRRKünstlers wie Evgenii Kacman, dessen Gemälde "Spitzenklöpplerinnen“ (1928) an das Genrebild „Mutter und Schwester des Künstlers“ (1866) von Hans Thoma erinnert. An der Stelle der vorlesenden Schwester sitzt die junge Frau mit rotem Kopftuch, die den Typus der lesenden Arbeiterin verkörpert. Am 15. Juli 1929 beschloss der Rat der Volkskommissare, Künstler und Schriftsteller in Kolchosen und Fabriken abzukommandieren. Aber auch sieben Jahre nach Arvatovs Kritik dominierte die bürgerliche, die exotische Sicht auf den Produktionsalltag.

Unter diesem Druck der tatsächlichen Verhältnisse in den Fabriken und auf dem Lande (Analphabetentum und sozioökonomischer Rückständigkeit) ging die Avantgarde an den WChUTEMAS von der Analyse ihrer künstlerischen Mittel in der Laboratoriumsphase des Konstruktivismus über zur Synthese einer umfassenden „ideologischen Konstruktivität, die eine bewußte Organisiertheit unseres Denkens in allen Lebensfragen zur Grundlage hat“ ${ }^{32}$ Entsprechend den Losungen „Organisiertheit, Planmäßigkeit, Rationalisierung“ des Ersten Fünfjahrplans, mit dem eine nachgeholte und damit im Wettbewerb mit den kapitalistischen Ländern zwangsläufig forcierte Industrialisierung eingeleitet wurde, bezeichnete der Begriff „Konstruktivität“ jetzt die neue Funktionsbestimmung der Kunst als Mittel zur Umgestaltung der „PsychoIdeologie“ des Proletariats. Viktor Schklowski erklärte am 21.08.1934 auf dem bereits erwähnten Ersten Allunionskongreß der Sowjetschriftsteller: „Wir [...] LEF-Leute (Linke Front der Künste) [...] machten solche Konstruktionen, daß sie zu Unkonstruktionen wurden. Wir unterschätzten das Menschliche und das Gesamtmenschliche der Revolution [...].““33

Arbeiten von Alexander Deineka zwischen 1925 und 1935 zeigen den Wechsel von einer massenwirksamen operativen Kunst, die die komplexe Realität nicht in eine homogene Bildstruktur presst und in ihrem typischen Ausdruck repräsentiert, sondern argumentierend die Realität aus dem vertrauten Wahrnehmungszusammenhang herauslöst und als sich verändernde, bzw. veränderbare zeigt, zu einer traditionellen Genremalerei, die wieder an den individuellen Betrachter appelliert.

Deineka, 1899 in Kursk geboren, setzte sich 1924 mit einer Gruppe Gleichgesinnter von den Produktionskünstlern mit seinem Bekenntnis zur Malerei (später Wandmalerei) und zur publizistischen Kunst (Zeitschriftengrafik, Plakate) ab. 1925 gründeten sie gemeinsam die „Gesellschaft der Staffeleimaler" (OST). 1928 wurde er Mitglied der neu gegründeten Konstruktivistenvereinigung „Oktober“, die im Sinne einer Synthese der 
Künste unter der Vorherrschaft der Architektur das Gesamtkunstwerk einer kommunistischen Gesellschaft schaffen wollten. Neben Architekten, Filmemachern und Polygraphen waren hier auch die Maler der OST vereinigt. Sie wollten eine aktivistische, ins Leben eingreifende Kunst, keine subjektiven Ich-Ergüsse. Aus Deinekas publizistischer Phase stammt die Grafik „Die Macht der Sowjets“ für die Zeitschrift „Bezboschnik“ (Der Gottlose), Nr. 1, 1927. Mit wenigen Versatzstücken (Säule, Bogenfenster) wird der Ort der tagenden Sowjets als zaristisches, der alten Gesellschaft zugehöriges Gebäude bezeichnet. Doch das dunkle Gewölbe erfüllen über den Köpfen der Delegierten die neuen Losungen der Sowjetmacht: „Industrialisierung, Kooperative, Reduzierung der Preise!, handeln wir ökonomisch!“ Aus den Stuhlreihen steigt die Schrift auf: „Für den Kampf gegen Bürokraten, Kulaken und Popen!“ Den Zenit des Raumes schmückt das Wort „Lenin“, darunter „Sowjetmacht“ und „Rote Armee“. Deineka gibt nicht nur das Abbild einer stummen Menge wieder, sondern versucht, die Inhalte der Debatte als Handlungsaufforderungen an den Betrachter weiterzugeben. Präsidium und Delegierte befinden sich noch auf der gleichen Ebene. Auch der Redner wird gegenüber seinen Zuhörern nicht erhöht. Der Betrachter sieht seinen Repräsentanten ins Gesicht und nicht dem Agitator, wie es dem konventionellen Schema entsprochen hätte.Dagegen vollzieht A. M. Gerassimows Gemälde „Hymne auf den Oktober“, 1942, den Bruch zwischen der ,Avantgarde“ auf der Bühne und den Massen im Parkett des Bolschoitheaters, in dem kein Sowjet tagt, sondern die Festversammlung der Deputierten aller Parteiorganisationen Moskaus zum 25. Jahrestag der Oktoberrevolution am 6. November 1942. Sie findet statt unter der Porträtbüste Lenins als Symbol eines zum zeitlosen Prinzip erstarrten Leninismus. Die Perspektive hat sich verkehrt. Die Zuschauer schauen gebannt auf Stalin als Redner, der wie ein Schauspieler im Scheinwerferlicht steht. Das Fußvolk im Parkett und die durch bessere Kleidung gekennzeichnete Intelligenzia auf den Rängen geben ein zutreffendes Bild der stalinistischen Ständegesellschaft.

Die von oben angeordnete zweite und endgültige Kultur-Revolution, ein Bürgerkrieg gegen die eigene Bevölkerung, begann nach Stalins 50. Geburtstag im Dezember 1929. Aus diesem Anlass wurde ihm der bisher Lenin vorbehaltene Titel eines ,Woshd' (Führers) verliehen. Der bisher eher unauffällig agierende, uncharismatische Stalin mußte in der neuen Rolle seine Person mit der Geschichte der Partei Lenins identifizieren. Zunehmend verdrängt er jetzt in der Massenagitation den Kopf Lenins. ${ }^{34}$ Auf dem Plakat „Unter Lenins Fahne für den Aufbau des Sozialismus“ von Gustav Klucis von 1930 ist noch die Hälfte von Stalins Kopf von Lenin verdeckt, aber er tritt doch deutlich aus seinem Schatten heraus. Bei A. Gerassimow Gemälde „Stalins Rechenschaftsbericht auf dem 16. Parteitag“" von 1933 repräsentiert er bildfüllend in weißer Uniform (siehe „Wir").

Walter Ulbricht übernahm das Modell der sowjetischen Kulturrevolution 1959 im Maßstab der DDR. Alfred Kurella, 1955 aus der SU zunächst nach Leipzig als Leiter des Literaturinstituts zurückgekehrt, wurde auf der 33. ZK-Tagung im Oktober 1957 zum Leiter der dort gegründeten „Kommission für Fragen der Kultur beim Politbüro der SED“ und damit zum allmächtigen Kulturpolitiker, bis ihn, Ende 1962, Kurt Hager ablöste.Das geschah als Reaktion auf die Ereignisse in Ungarn 1956. Das Jahr 1957 stand im Zeichen der unerbittlichen Repression gegen alle Sympathisanten von Reformen. Unter den über 4000 verhafteten „Staatsfeinden“ waren u.a. der Leipziger Schriftsteller Erich Loest und der Philsoph Wolfgang Harich. Die Prozesse dienten als willkommener Vorwand, die Formalismuskampagne von 1951/1953 unter den neuen Schlagworten 
„ästhetischer Revisionismus“ und „Dekadenz“ wieder aufzunehmen. Kurz nach seiner Ernennung nutzte Kurella die erste Kulturkonferenz der SED am 23. bis 24. Oktober 1957 in der Parteihochschule „Karl Marx“ in Ost-Berlin für die Propagierung einer Kulturrevolution nach sowjetischem Vorbild.

Alfred Kurella als Chefplaner dieser neuen Kulturrevolution, die mit der Bitterfelder Konferenz im April 1959 ihre praktische Umsetzung erfuhr, und unmittelbar an den „Kulturfeldzug“ in der Sowjetunion 1929 anschloss, war von 1927 bis 1929 bereits Leiter der Hauptverwaltung für Schöne Kunst und Literatur im Volkskommissariat für Volksaufklärung unter Anatoli Lunatscharski sowie Redakteur für Literatur und Kunst der Komsomolskaja Prawda gewesen. In diesen Positionen nahm er entscheidenden Einfluss auf die Kunstdebatten und die Kulturrevolution während des Ersten Fünfjahrplanes ab 1929. Mit dem ungarischen Kunstkritiker und Regisseur Janos Máca und dem sowjetischen Kunsttheoretiker A. Michajlov verfasste er die Deklaration vom 3. Juni 1928 der bereits erwähnten Avantgardegruppe „Oktober“. Darin polemisierten die drei Autoren gegen „den spießerhaften Realismus der Epigonen“, gegen „den Realismus der stagnierenden individuellen Lebensweise, den passiv-beschaulichen, der die Wirklichkeit lediglich fruchtlos kopiert, der die alte Lebensweise verherrlicht und kanonisiert, der die Energien fesselt und den Kulturwillen des ungefestigten Proletariats schwächt" ${ }^{\text {" }}{ }^{35}$

Doch niemand in der DDR wusste von Kurellas Polemiken gegen diesen GenreRealismus konservativer Gruppierungen wie die AChRR, die ab 1932 zum Vorbild für den S.R. erklärt wurden. Kurella sorgte dafür, dass nichts davon in der DDR veröffentlicht wurde. Inzwischen lobte der alternde sozialistische Humanist Kurella die Genremalerei eines Heinrich Witz (vgl. „Der neue Anfang“, 1959) und die altmeisterliche Lasurtechnik eines Werner Tübke. Auf der einzigen Sitzung der Kulturkommission am 19. Oktober 1959, die ausschließlich Fragen der Bildenden Kunst behandelte, kündigt Kurella an, im Frühjahr 1960 auf dem Gebiet der bildenden Kunst etwas ähnliches machen „wie (ein Jahr davor, E. G.) in Bitterfeld auf dem Gebiet der Literatur $[. . .]^{{ }^{* 36}}$ Ziel sei, eine große Bewegung „Künstler und Volk sind eins“ zu organisieren (Bl. 211). Insgesamt sei die führende Rolle der Partei auf dem Gebiet der Malerei zwischen 1954 und 1958 nicht gesichert gewesen. Das Auftragswesen sei zu reorganisieren mit dem Ziel, „es zu einem Mittel der aktiven Kunstpolitik zu verwandeln". Die Partei erhoffte sich von der strengen Schule der Betriebe unter den wachsamen Augen der Werktätigen das Heranwachsen eines neuen Künstler-Typus.Die Künstler als ,bürgerlichen' Einzelgänger und Individualisten sollen jetzt auf dem „Bitterfelder Weg“ von den Brigaden diszipliniert und dem Einfluss der Funktionäre gefügig gemacht werden.

Gegen diese Sowjetisierung der Kunst im Sinne eines zeitlosen Akademismus wehrten sich die Künstler, vor allem die Remigranten, die in der Weimarer Republik einem anderen Begriff von Realismus verpflichtet waren, der nicht umsonst auch Verismus (von veritas=Wahrheit) genannt wurde. Auf einer Vorstandssitzung des VBKD zum Thema „Neuer Kurs und die Bildenden Künstler“ erklärte Lea Grundig im November 1953 empört: „Vor allen Dingen unserer Generation, zu der Kollege Cremer gehört, [...] hat man wirklich die Füße abgeschnitten, mit denen wir auf dem Boden standen, und man hat von uns verlangt, wir sollten unser gesamtes, bis dahin geschaffenes Werk für begraben erklären und völlig von neuem anfangen. Sie und ihre Künstlerkollegen in der ASSO haben doch fest an der Seite der kommunistischen Partei mit den Mitteln der 
bildenden Kunst gekämpft. Das sei jetzt alles ,Formalismus ${ }^{6}{ }^{37}$ Schon 1949 ist Lea Grundig dennoch bereit, ihre differenzierte Porträtkunst in der Tradition des Verismus und der Neuen Sachlichkeit z.B. einzusetzen für vorbildliche Arbeiter wie den „Koksmeister Oskar Schädlich“, Aktivist im Steinkohlenwerk „Karl Marx“ in Zwickau (1951).

31 Im „Kampf gegen die Dekadenz“ soll die Arbeiterklasse in der DDR mobilisiert und motiviert werden, Westdeutschland ,auf allen wichtigen Gebieten der Versorgung der Bevölkerung mit Lebensmitteln und Konsumgütern“ bis 1961 einzuholen. ${ }^{38}$ Den Elan für diese außerordentliche Anstrengung, zu der alle materiellen Voraussetzungen fehlen, soll die Kulturpolitik organisieren. Deshalb fordert Walter Ulbricht in seiner Rede auf dem V.Parteitag der SED im Juli 1958 die Arbeiterklasse auf, auch „die Höhen der Kultur“ des emanzipierten Bürgertums der Goethe-Zeit zu „erstürmen und von ihnen Besitz (zu) ergreifen “. ${ }^{39}$ Der bürgerliche Humanismus als die geglückte Einheit von Wahrheit, Schönheit und Harmonie gilt in der DDR bis zu ihrem Ende als normativer Maßstab „sozialistisch-humanistischer“ Kunstwerke. Diese Werte werden von der „aufsteigenden“ Arbeiterklasse, die in eine Menschengemeinschaft sich verwandle, bewahrt und vollendet. Die Kulturpolitik der SED orientiert sich am aristokratischen Ideal der „allseits entwickelten Persönlichkeit“. Solange sich soziales Schöpfertum der „neuen Eigentümer“ noch nicht am Arbeitsplatz entfalten kann, will man im „kulturvollen Freizeitverhalten“ nach Feierabend einen Ausgleich schaffen. In seiner Rede zur 2. Bitterfelder Konferenz sprach Ulbricht vom ewig faustischen Wollen, diesem Streben „nach voller Entfaltung der Persönlichkeit“, das sein Zentrum in der DDR gefunden habe. Die Faust-Figur verkörperte aber auch für Ulbricht die Kontinuität bürgerlicher Emanzipation als rücksichtslose Beherrschung und Ausbeutung der Natur durch die Entfesselung der „Wissenschaftlich-Technischen Revolution“.

32 Alfred Kurella verengt dieses kulturelle Erbe des bürgerlichen Humanismus in der Malerei auf den Klassizismus als Stilform des deutschen Idealismus. Nicht Dürer, nicht Menzel oder Leibl sind Kurellas Ideal, sondern der Spätnazarener Peter Cornelius. In einem Zwischenruf fordert er: „Wie Cornelius malen!“40 Diese Bemerkung von Alfred Kurella verdeutlicht die Nähe der von ihm propagierten Vorstellung des Sozialistischen Realismus zum Nazarenertum der Lukasbrüder, die eine Symbiose von akademischem Klassizismus und Nationalromantik zu einer konservativen Ideenkunst erstrebten. Im Sinne einer Nationalpädagogik wollten diese mit ihrer Kunst, wie in den fünfziger Jahren Alfred Kurella und die SED, die Einheit von Kunst und Volk bzw. Nation in kollektiver, nach festgelegten Regeln organisierter Arbeit verwirklichen. Die Ästhetik des deutschen Frühklassizismus, welche die Einheit von Zeit, Ort und Raum und den "fruchtbaren Augenblick“ herausstellte, wird von Kulturfunktionären wie Gerhard Winkler zum normativen Vorbild der Ästhetik des Sozialistischen Realismus erklärt. ${ }^{41}$

So konnte Kurellas Versuch, die große sowjetische Kulturrevolution von 1929 in der DDR des Jahres 1959 zu wiederholen, nur zu einer Travestie seines Vorbildes werden.

Entsprechend dieser idealistischen Position des Sozialistischen Realismus zählt die Darstellung der gefährlichen, gesundheitsschädigenden, oft unter haarsträubenden Sicherheitsbedingungen geleisteten Arbeit unter Tage nicht zu den ,fruchtbaren Momenten' der Malerei. Dies tut der Roman Rummelplatz von Werner Bräunig, ein 31jähriger schreibender Arbeiter am Literaturinstitut, der 1953 in den radioaktiv verstrahlten Schächte des Uranbergbaus der Wismut gearbeitet hatte. Von ihm stammt die Losung der Bitterfelder Konferenz „Greif zur Feder, Kumpel“. Sein Roman über die 
Aufbaujahre der DDR und seine Erfahrungen bei der Wismut zwischen 1949 und dem 17. Juni 1953 stehen im Dezember 1965 während des 11. ZK-Plenums im Zentrum der Kritik, die sich auf den Vorabdruck in der Zeitschrift Neue deutsche Literatur (Heft 10, 1965, S. 7-29) bezieht. Obwohl Anna Seghers, Christa Wolf und Fritz Erpenbeck das Buch verteidigten, kann der in der NDL für das kommende Jahr angekündigte Roman erst 40 Jahre später $2007 \mathrm{im}$ Aufbau-Verlag erscheinen. Bräunig gerät nach dem negativen Urteil über seinen Roman in Vergessenheit, verfällt dem Alkohol und stirbt 1976 mit 42 Jahren. Christa Wolf aber wird auf diesem „Kahlschlagplenum“ im Dezember 1965 klar, „daß die Verbindung der Künstler mit den Betrieben dazu führte, daß sie realistisch sahen, was dort los war, daß sie Freundschaften mit Arbeitern, mit Betriebsleitern und mit Leuten anderer Berufe knüpften und daß sie Bescheid zu wissen begannen auch über die ökonomische Realität in diesem Land: Da, genau an diesem Punkt, wurde die Bitterfelder Konferenz, wurden die Möglichkeiten, die sie uns eröffnet hatte, ganz rigoros beschnitten. [...] Damit wurde also die Möglichkeit zur Einmischung durch Kunst, die wir vehement ergriffen hatten [...] gekippt“ “. ${ }^{42}$

Zwar wuchs in der Periode des „Neuen ökonomischen Systems der Planung und Leitung" (NÖSPL) in den 60er Jahren die Einsicht, dass die sozialistische Produktion „den selbständig denkenden, sicher reagierenden, wissenschaftlich gebildeten [...] arbeitenden Menschen “43 benötigt. Die SED erkannte, dass das autoritäre, auf Gehorsam, Unterordnung und Anpassung geeichte System mit seinem stalinistischen Misstrauen gegen jede Art von selbständigem Denken in einen grundsätzlichen Widerspruch zu den Erfordernissen der wissenschaftlich-technischen Revolution stand. Der Kunstwissenschaftler Peter H. Feist forderte entsprechend dem wachsenden Anteil intelligenzintensiver Produkte wie Elektronenrechner statt Schaufelstiele eine „intelligenzintensive Kunst“ ${ }^{\text {“ }}{ }^{4}$ „Es galt, etwas nicht unmittelbar sichtbar Werdendes anschaulich zu machen, nämlich geistige Arbeit - Planen, Denken, Entwerfen, Entscheiden, Verantworten. “ ${ }^{45}$ Die Perspektive des Planers und Leiters wurde erstmals in dem Gemälde "Schachspieler“, 1964, von Willi Neubert thematisiert. Mit dem "Chemiearbeiter am Schaltpult", 1968, gelang dann Willi Sitte ein Musterbild der gewünschten „intelligenzintensiven“ Kunst. Der Maler bringt sein Publikum in einen direkten Dialog mit dem Kunstwerk über den Kunstgriff einer transparent gemachten Schaltwand, durch die hindurch der Betrachter auf die höchste Konzentration und Verantwortungsbewusstsein zum Ausdruck bringenden Gesichtszüge des Chemiearbeiters schaut wie auf sein alter ego im Spiegel.Die Kunstwissenschaftler sprachen im Zusammenhang mit dem Chemiearbeiter vom „dialogischen Bild“ , ${ }^{46}$ das den mitdenkenden, aktiven Betrachter als Partner des Künstlers ernst nehmen sollte. Die dominant weit in den Vordergrund ausgestreckten Hände an den Schalthebeln komplizierter Produktionsabläufe im Zentrum eines „sozialistischen Chemie-Giganten“ überhöhen den Industriearbeiter zum Demiurgen: Auf das "Götterbild, das Jahrtausende lang die Kunst beherrschte“, erhebt jetzt der Arbeiter Anspruch.

Im Betriebsalltag der „sozialistischen Rationalisierung“ dagegen öffnete sich die Schere zwischen der immer anspruchsvolleren Tätigkeit der Ingenieure und der rezeptiven Vereinfachung der Arbeit bei den Werktätigen. Die ideologische Funktion des Sozialistischen Realismus lag in der Suggestion, jeder Facharbeiter könne in der sozialistischen Menschengemeinschaft komplexe Produktionsprozesse allein steuern und unterscheide sich dank forcierter Qualifikationsmaßnahmen nicht wesentlich von einem Wissenschaftler. Aber gerade das ist ja die Funktion des Sozialistischen Realismus, den „sozialistischen Charakter der Arbeit und unserer ganzen Gesellschaft 
in jedem Bereich und an jedem Arbeitsplatz auch subjektiv und anschaulich erleben “ $\mathrm{zu}$ lassen. ${ }^{47}$

Die „2. Bitterfelder Konferenz“ (24./25.04.1964) zog Konsequenzen aus der Kritik der Künstler an ihrer Bevormundung und Gängelung durch die Partei: Die Kulturfunktionäre nahmen Abschied von der Vorstellung, Kunst und Leben ineinander aufgehen $\mathrm{zu}$ lassen, und setzten wieder auf den Berufskünstler und seine Propagandafunktion, von der Walter Ulbricht in seiner Rede sagte: „Wir sehen in den Künstlern und Kulturschaffenden nicht bloße ,Fachleute für eine sinnvolle Feierabendgestaltung‘. Wir messen ihnen eine weitaus größere gesellschaftliche Rolle zu. Ich sage offen: wie gut und wie schnell es bei uns vorwärts geht, das hängt in einem hohen Grade von ihrer zielstrebigen und guten Arbeit ab." ${ }^{48}$

Gegen den, wie es der Maler Gerhard Richter es ausdrückte, „verbrecherischen Idealismus“ der Kommunisten schrieb Georg Büchner um 1835 in seiner Erzählung „Lenz":

Das Gefühl, dass, was geschaffen sei, Leben habe, [...] sei das einzige Kriterium in Kunstsachen. [...] Da wollte man idealistische Gestalten, aber alles, was ich davon gesehen, sind Holzpuppen. Dieser Idealismus ist die schmählichste Verachtung der menschlichen Natur. ${ }^{49}$

Lea Grundigs Zeichnungen von Arbeitern und Kindern, z.B. „Frauen in der Qualifikation“ (1967) oder „Drei von der Brigade“ sind nicht idealisiert, zeigen skeptische, aufmerksame Frauen, selbstbewusst und distanziert. Sie knüpfen an ihre Zeichnungen, z.B. von proletarischen Kindern und Arbeiterfrauen von 1930 an und folgen Büchners Devise:

Man muß die Menschen lieben, um in das eigentümliche Wesen jedes einzudringen. Es darf einem keiner zu gering, keiner zu hässlich sein, erst dann kann man sie verstehen; das unbedeutendste Gesicht macht einen tieferen Eindruck als die bloße Empfindung des Schönen [...]. Der Dichter und Bildende ist mir der liebste, der mir die Natur am wirklichsten gibt, so daß ich über seinem Gebild fühle [...]. ${ }^{50}$

\section{NOTES}

1. B. Groys, „Konstruktion als Subtraktion“, in H Gaßner, K. Kopanski und K. Stengel (Hrsg.), Die Konstruktion der Utopie. Ästhetische Avantgarde und politische Utopie in der 20er Jahren, Marburg, Jonas Verlag, 1992, S. 74.

2. A. Kurella, „Erfahrungen und Probleme sozialistischer Kulturarbeit“, in Kulturkonferenz 1960, Protokoll, Berlin-DDR, Dietz Verlag, 1960, S. 15.

3. Pablo Picasso lieferte mit der Lithographie einer Taube, die Louis Aragon 1949 auswählte, das Symbol der kommunistischen Friedensbewegung.

4. M. Gorki, „Über sowjetische Literatur“ (1. Sitzung am 17.08.1934), Zit.n. H.-J. Schmitt und G. Schramm (Hrsg.), Dokumente zum. I. Allunionskongreß der Sowjetschriftsteller, Frankfurt am Main, 1974 , S. 81.

5. J. Immendorff, Hier und Jetzt: Das tun, was zu tun ist, Köln-New York, König, 1973, S. 35. 
6. Imi Knoebel im Gespräch mit Johannes Stüttgen, zit.n. IMI Knoebel Retrospektive 1968-1996, Ausst.-Kat. Haus der Kunst, München, 1996, S. 51.

7. Veröffentlicht am 28.03.1918 in der Zeitung Anarchija.

8. A. A. Bogdanow, Der rote Stern. Ein utopischer Roman, Darmstadt und Neuwied, Hermann Luchteehand Verlag, 1982. Deutsche Erstausgabe: Verlag der Jugendinternationale, BerlinSchöneberg, 1923, S. 68ff.

9. Eigentlich Alexander Alexandrowitsch Malinowskij 1873-1928. Zwischen 1906 und 1910 galt er als Konkurrent Lenins um die Führung des bolschewistischen Flügels der russischen Sozialdemokratie. Vgl. D. Grille, Lenins Rivale - Bogdanov und seine Philosophie, Köln, Verlag Wissenschaft und Plitik, 1966.

10. A. A. Bogdanow, Allgemeine Organisationslehre, Tektologie, Bd. I, Berlin 1926, S.10. Zit. n. Proletarische Kultur (Proletkult), Ästhetik und Kommunikation, Heft 5/6, Februar 1972, Jg. 2, S. 94.

11. L. Trotzkij, Literatur und Revolution, Berlin, 1968, S. 161.

12. Nadeschda Mandelstam spricht in ihrer Autobiographie Das Jahrhundert der Wölfe von der "Periode der Umwertung der Werte“ und nennt das 37. Kapitel „Die Umwertung der Werte“ (Frankfurt am Main, 1991, S. 203).

13. L. Trotzkij, a.a.O., S. 85.

14. Ebd., S. $212 f$.

15. L. Trotzkij, a.a.O., S. 214f. Auch Ernst Jünger beschwor die Vision des kollektiven Übermenschen als Typus des Kriegers (1930) und Arbeiters (1932): „Das ist der neue Mensch [...]. Baumeister werden sie sein auf den zertrümmerten Fundamenten der Welt. [...] Das ist der vollkommene Zusammenbruch des Individualismus, es ist der absolute Bankrott des humanitären Denkens.“ (Der Krieger)

16. Stalin interpretiert 1930 Lenins Theorie über das Absterben des Staates (Staat und Revolution, 1917) dahingehend, dass dieses Absterben „nicht durch Schwächung der Staatsmacht erfolgen, sondern durch ihre maximale Verstärkung“ zu erreichen sei, „die notwendig ist, um die Überreste der sterbenden Klassen zu vernichten [...]“. (J.W. Stalin, Werke, Bd. 12, Berlin/DDR, 1950, S. 189)

17. Vgl. H. Gaßner und E. Gillen, Vom utopischen Ordnungsentwurf zur Versöhnungsideologie im ästhetischen Schein. Beispiele sowjetischer Kunst zwischen dem 1. Fünfjahrplan und der Verfassungskampagne 1936/1937. In Agitation zum Glück. Sowjetische Kunst der Stalinzeit, Ausst.Kat. documenta Archiv Kassel, Bremen, Temmen, 1994, S. 27-59.

18. N. Mandelstam, Das Jahrhundert der Wölfe, Frankfurt am Main, 1991, S. 56.

19. Die beiden Bände seiner Tektologie erschienen 1913 und 1917, deutsch: Berlin 1926 und 1928.

20. Vgl. den u.a. von Bogdanow herausgegebenen Sammelband „Umrisse einer Philosophie des Kollektivismus“, 1909, in dem auch der Aufsatz von Maxim Gorki „Die Zerstörung der Persönlichkeit" enthalten war.

21. Vgl. H.Gaßner, „Aleksandr Rodschenko - Konstruktivistische Fotografie“, in Ders., Rodtschenko Fotografien, München, Schirmer-Mosel, 1982, S. 26, Anm. 94, S. 125.

22. Ebd., S. 27.

23. Zur Umformung der Individualisten in Kollektivisten braucht das Proletariat „seine eigene Klassenkunst. Der Geist dieser Kunst ist der Arbeitskollektivismus; sie nimmt die Welt wahr und widerspiegelt sie vom Standpunkt des Arbeitskollektivismus“. (A. A. Bogdanow, Resolution zur ersten Allunionskonferenz der proletarischen Kultur- und Aufklärungsorganisation, September 1919, zit.n. D. Grille, a.a.O., S. 185f.)

24. S. Tretjakow, „Woher und wohin. Perspektiven des Futurismus“, LEF, Nr. 1, MoskauPetrograd 1923, S. 195, zit.n. B. Groys, Gesamtkunstwerk Stalin, München 1988, S. 43.

25. Geboren 1884 in Lebedjan, 1937 in Paris gestorben. Er beteiligte sich bereits 1905 an politischen Aktionen: und war zeitweise Mitglied in der bolschewistischen Fraktion der Sozialdemokratischen Arbeiterpartei Rußlands, wurde verhaftet, verbannt und lebte in der 
Illegalität. Nach seiner erneuten Verhaftung im August 1922 weigerte er sich, Russland zu verlassen und protestierte erfolgreich gegen den Ausweisungsbeschluss. (Vgl. K. Kasper, „Nachwort", in J. Samjatin, Werke, Band IV, Leipzig und Weimar, 1991, S. 154-189.)

26. „Wir" war der pogrammatische Titel zahlreicher Gedichte von Proletkultdichtern wie Wladimir Kirillow und Futuristen wie Wladimir Majakowskij. Über dem Eingang der WChUTEMAS stand die Losung „Wir/Boten eines neuen Glaubens/an die Schönheit, die stählern ertönt..."(W. Majakowskij)

27. Der Roman galt sofort als antisowjetische Schmähschrift und konnte erst 1988 in der Sowjetunion erscheinen. 1925 erstmals in England veröffentlicht, wurde er zum Prototyp der antitotalitären, negativen Utopien von Aldous Huxley („Schöne neue Welt", 1932) und George Orwell („Farm der Tiere“, 1945 und „1984“, 1949).

28. A. Gastev, „Geradebiegen des Volkes“ (Narodnaja vypravka), in Prawda, Moskau, 1922, Nr. 128, zit.n. der deutschen Übersetzung in alternative, Heft 122/123, Berlin, 1978, S. 242-246, hier S. 245.

29. A. Rodtschenko, „Linija“, Moskau, 1921, in russischer Sprache unveröffentlichtes Typoskript eines Vortags für das INChUK (Institut für Künstlerische Kultur). Zit.n. der deutschen Übersetzung, in H.Gaßner und E.Gillen (Hrsg.), Zwischen Revolutionskunst und Sozialistischem Realismus. Dokumente und Kommentare, Köln, DuMont Verlag, 1979, S. 116.

30. V. Kataev, Vremja, vpered, 1932, Roman, zit.n. H. Gaßner, Rodtschenko Fotografien, München, 1982, S. 107, Anm. 498.

31. „Die AChRR in der Fabrik“, Kunstleben, Nr. 30, 1925, H. Gassner und E. Gillen, a.a.O., S. 416.

32. „Ein Kunstwerk kann konstruktivistisch und gleichzeitig aber nicht konstruktiv sein - und umgekehrt: konstruktiv sein heißt nicht ,konstruktivistisch“ sein.“ („Die Kunst der UdSSR und die Aufgaben der Künstler. Diskussion in der Kommunistischen Akademie“. I. Maca, Schlußwort, März 1928. Zit.n. H. Gaßner und E. Gillen, a.a.O., S. 495-500, hier S. 498.)

33. H.-J. Schmitt und G. Schramm (Hrsg.), Sozialistische Realismuskonzeptionen. Dokumente zum I. Allunionskongreß der Sowjetschrifsteller, Frankfurt am Main, 1974, S. 95.

34. Kat. Klucis, VI. 37, S. 375.

35. Zit.n. H. Gaßner und E. Gillen (Hrsg.), Von der Revolutionskunst zum Sozialistischen Realismus, Köln, DuMont Verlag, 1979, S. 181.

36. SAPMO-BArch DY 30/IV 2/906/53, Bl. 176.

37. L. Grundig, Rede auf der außerordentlichen Vorstandssitzung des VBKD am 14.11.1953. In Neuer Kurs und die bildenden Künstler. Hrsg. vom Verband Bildender Künstler Deutschlands, Zentralleitung, Dresden o.J. [1953], S. 142.

38. Interview mit Walter Ulbricht im ND vom 21.08.1959. Zit.n. A. Mitter und S. Wolle, Untergang auf Raten, S. 301.

39. W. Ulbricht, „Die sozialistische Umwälzung der Ideologie und der Kultur“, in Protokollband zum V. Parteitag der SED: Für den Sieg der sozialistischen Revolution auf dem Gebiet der Kultur, Berlin, 1958, S. 3-42.

40. Nach dem Bruch mit dem bayrischen König Ludwig I. folgte Cornelius (1783-1867) einem Ruf Friedrich Wilhelms IV. nach Berlin. Der „Romantiker auf dem Thron“ sah in Cornelius den Künstlertypus, der seinen Vorstellungen von der königlichen Mittlerstellung zwischen Gott und dem Volk (im Sinne der Einheit von Thron und Altar) den angemessenen künstlerischpädagogischen Ausdruck geben könne.

41. Gerhard Winkler, der 1961 seine Dissertation am Institut für Gesellschaftswissenschaften beim ZK der SED, der Parteihochschule, über die „Bedeutung von Lessings Schrift Laokoon für die deutsche sozialistische nationale bildende Kunst" schreibt, behandelt darin auf über 40 Seiten die Gemälde von Heinrich Witz als Musterbilder im Sinne von Winckelmann und Lessing. Nach seinem Studium wird er Kulturbeauftragter der SED-Gebietsleitung bei der SDAG Wismut und 
arrangiert in dieser Funktion eine Begegnung zwischen dem Künstler Heinrich Witz mit zwei Wismut-Brigaden.

42. C. Wolf, Erinnerungsbericht, in Kahlschlag, S. 344-354. Hier: 347, 350f. In diesem Sammelband ist auch ihre Rede auf dem Plenum abgedruckt, von der die ND-Beilage zum 11. Plenum. Aus der Diskussion vom 19.12.1965, S. A 12, nur Auszüge brachte.

43. F. Staufenbiel, Kultur heute - für morgen. Theoretische Probleme unserer Kultur und ihre Beziehung zur technischen Revolution, Berlin, Deutscher Verlag der Wissenschaften, 1966, S. 98. Vgl. auch F. Staufenbiel, „Die technische Revolution und der Mensch unserer Zeit“, Bildende Kunst, Heft 2, 1965. Mit diesem Artikel eröffnete die Zeitschrift eine neue Diskussionsreihe unter dem Titel „Mensch - Technik“.

44. P. H. Feist, „Muß unsere Kunst intelligenzintensiv sein?“, Bildende Kunst, Heft 8, 1966, S. 435. Vgl. auch E. Gillen, „Herkules am Schaltpult. Arbeiterdarstellungen 1951 bis 1977“, insbesondere das Kapitel „Intelligenzintensive Kunst aus der Perspektive des Planers und Leiters“, in AufbruchAnkunft-Ausbruch. 30 Jahre DDR-Kunst und Literatur, Schriftenreihe DDR-Kultur 1, Berlin, Paul-LöbeInstitut, 1981, S. 36-39.

45. P. H. Feist, „Der Mensch und seine Werke“, Dezennium 2, Dresden, 1972, S. 15.

46. Vgl. P. H. Feist, „Das Kunstwerk als Gesprächspartner“, Bildende Kunst, Heft 3, 1974, S. $122 \mathrm{ff}$.

47. Beiträge zur sozialistischen Arbeitskultur, Berlin-DDR, Tribüne, 1973, S. 90.

48. „Über die Entwicklung einer volksverbundenen sozialistischen Nationalkultur“, Rede Walter Ulbrichts auf der 2. Bitterfelder Konferenz, 24./25.04.1964, in Neues Deutschland, 28.04.1964. Zit.n. Kunstkombinat, Berlin, 1990, S. 56.

49. G. Büchner, „Lenz“, in Werke und Briefe, München, 1965, S. 71f.

50. G. Büchner, ebd., S. $72 f$.

\section{RÉSUMÉS}

Mit dem Programm des „Bitterfelder Weges“, benannt nach der „I. Bitterfelder Konferenz“ im April 1959 im Kulturhaus auf dem Gelände des VEB Elektrochemisches Kombinat Bitterfeld, fordert die SED von den Künstlern, die „Einheit von Kunst und Leben“ sowie die Überwindung der „Kluft zwischen Kunst und Volk“ durch die „Volkstümlichkeit“ ihrer Kunst und die Förderung der Laienkunst („Bildnerisches Volksschaffen“) unter Anleitung der Berufskünstler zu realisieren. „Auf diese Weise [...] wird die Trennung von Kunst und Leben bei uns in der DDR ein für allemal überwunden werden.“ Die Künstler sollten als Zirkelleiter die Arbeiter künstlerisch ausbilden, um sie so im Sinne des „sozialistischen Humanismus“ zu „,neuen Menschen“, die alle ihre Fähigkeiten entfalten können, zu machen. Damit sollte die kapitalistische Ausbeutung der Arbeiter durch einseitige Arbeit am Fließband überwunden werden. Der Appell, die Trennung zwischen Kunst und Leben aufzuheben, erinnert an die Manifeste der deutschen Expressionisten und Aktionisten nach dem Ersten Weltkrieg sowie der sowjetischen Avantgarde während der Kulturrevolution in den 20er Jahren. Die Künstler als ,bürgerlichen“ Einzelgänger und Individualisten sollen auf dem „Bitterfelder Weg“ von den Arbeiterkollektiven diszipliniert und dem Einfluss der Partei-Funktionäre gefügig gemacht werden. Das Prinzip der Erziehung des Künstlers durch das Volk schlägt aber in sein Gegenteil um: Die Künstler solidarisieren sich mit den Arbeitern und ihrem Widerstand gegen schlechte Arbeitsbedingungen. Christa Wolf wird auf dem „Kahlschlagplenum“ im Dezember 1965 klar, „daß die Verbindung der Künstler mit den 
Betrieben dazu führte, daß sie realistisch sahen, was dort los war, daß sie Freundschaften mit Arbeitern, mit Betriebsleitern und mit Leuten anderer Berufe knüpften und daß sie Bescheid zu wissen begannen auch über die ökonomische Realität in diesem Land“. Daher wurde von der Partei diese Möglichkeit zur Einmischung durch Kunst, die von Künstlern und Schriftstellern vehement ergriffen worden ist, um 1965 wieder beendet. Es entstand aber in der Folge eine wirklich ,realistische‘ Kunst in der DDR, die den idealistischen und verlogenen „Sozialistische Realismus" überwand. Parallelen mit Ai Weiweis Rolle in China und dem Umgang mit ihm liegen auf der Hand.

En publiant son programme de la "voie de Bitterfeld», dont le nom se rapporte au premier congrès de Bitterfeld en avril 1959 dans la Maison de la Culture de l'entreprise du peuple (= nationalisée) qui porte le nom de combinat électrotechnique de Bitterfeld, le parti socialiste unifié est-allemand (= parti communiste) exige des artistes "d'unir l'art avec la vie », de combler « le profond fossé qui sépare l'art du peuple » en créant un art « populaire » et en promouvant un art « amateur » (« la création artistique populaire ») dirigé par des artistes professionnels. « Ainsi [...] sera une fois pour toute abolie dans notre RDA la séparation de l'art et de la vie. » Les artistes devaient donc animer des cercles où ils donneraient aux ouvriers une formation artistique en vue de les transformer, conformément à l'« humanisme socialiste », en " hommes nouveaux » capables d'épanouir toutes leurs potentialités. Ainsi serait dépassée l'exploitation capitaliste des ouvriers contraints à un travail à la chaîne unilatéral. L'appel à abolir la frontière entre art et vie fait penser aux manifestes des expressionnistes et «actionnistes " allemands après la première guerre mondiale et à l'avant-garde soviétique de la révolution culturelle des années 1920. Selon la «voie de Bitterfeld», les «collectifs" d'ouvriers devaient discipliner les individualistes bourgeois qu'étaient les artistes que l'influence des cadres du Parti devait enfin soumettre. Le principe de l'éducation de l'artiste par le peuple aboutit au contraire : les artistes se solidarisent avec la résistance des ouvriers aux mauvaises conditions de travail. Lors du fameux Plénum du Parti dit de la «table rase» de décembre 1965, l'écrivain Christa Wolf comprend "que les relations entre les artistes et les entreprises les conduisaient à voir la réalité de ce qui s'y passait et à nouer des amitiés avec les ouvriers, les directeurs et d'autres professions et à être en fin de compte très bien informés de la réalité économique du pays ». C'est pour cette raison que le Parti mit en 1965 un terme à cette possibilité d'agir ouverte à l'art que les artistes et les écrivains avaient si bien su exploiter. La conséquence de l'expérience fut la naissance en RDA d'un art « vraiment » réaliste, qui dépassa le « réalisme socialiste » idéalisateur et mensonger. Le parallèle avec le rôle d'Ai Weiwei en Chine et le traitement qui lui a été réservé s'impose.

\section{INDEX}

Mots-clés : URSS, RDA, arts visuels, réalisme socialiste, avant-garde, répression, révolution culturelle, voie de Bitterfeld

Schlüsselwörter : Sowjetunion, DDR, bildende Künste, Realistischer Sozialismus, Avantgarde, Repression, Kulturrevolution, Bitterfelder Weg

\section{AUTEUR}

ECKHARD GILLEN

Kulturprojekte Berlin 